Contents available at PubMed

Rev Invest Clin. 2018;70:211-6

\title{
DYSLIPIDEMIA IN MEXICO, A CALL FOR ACTION
}

\author{
Bernardette Rivas-Gomez ${ }^{1}$, Paloma Almeda-Valdés ${ }^{1,2}$, María Teresa Tussié-Luna $^{3}$ \\ and Carlos A. Aguilar-Salinas ${ }^{1,2,4}$
}

${ }^{1}$ Department of Endocrinology and Metabolism; ${ }^{2}$ Metabolic Disorders Research Unit; ${ }^{3}$ Molecular Biology and Genomic Medicine Unit, Instituto Nacional de Ciencias Médicas y Nutrición Salvador Zubirán, Mexico City; ${ }^{4}$ Tec Salud, Instituto Tecnológico y de Estudios Superiores de Monterrey, Monterrey, N.L., Mexico

\section{ABSTRACT}

The purpose of this manuscript is to highlight the peculiarities of the Mexican population regarding the clinical expression, genetics, and treatment of lipid disorders. Furthermore, it is a call for action to address the existing gaps in care and research of dyslipidemias. The Mexican Mestizos are highly susceptible to metabolic disorders (i.e., low high-density lipoprotein cholesterol concentrations, hypertriglyceridemia, abdominal obesity, and type 2 diabetes); these conditions are associated with ethnicspecific genetic variants. On the other hand, despite the high prevalence of dyslipidemia in Mexican adults, there is a lack of awareness of these conditions. The public is not informed about the need for screening and the potential benefit of the lipidlowering treatments. Underdiagnosis and undertreatment are two of the main challenges to be solved. Dyslipidemias are not among the priorities of the health systems for the prevention of cardiovascular disease; access to laboratory resources and medications is insufficient in primary care units despite the proven cost-benefit of the treatment of lipid disorders. Evidencebased public policies are needed to change the practice and allocation of assets to be capable of preventing cardiovascular diseases. Treatment of dyslipidemia should have a prominent role in any effort to decrease the number of preventable deaths caused by non-communicable diseases. (REV INVEST CLIN. 2018;70:211-6)

Key words: Dyslipidemia. Cholesterol. Triglycerides. High-density lipoprotein. Low-density lipoprotein. Mexico.

Corresponding author:

*Carlos A. Aguilar-Salinas

Departamento de Endocrinología y Metabolismo

Instituto Nacional de Ciencias Médicas

y Nutrición Salvador Zubirán

Vasco de Quiroga, 15

C.P. 14080, Mexico City, Mexico

E-mail: caguilarsalinas@yahoo.com

Received for publication: 02-05-2018

Approved for publication: 13-06-2018 doi: $10.24875 /$ RIC. 18002573 


\section{INTRODUCTION}

Dyslipidemias are common, multifactorial diseases that are major determinants of the cardiovascular risk. Genetic and environmental factors interact to determine the plasma lipid levels of a person and a population ${ }^{1}$. Its prevalence varies between countries and even through the years. Caucasians have higher cholesterol concentrations compared to those found in other ethnic groups, even if they live in the same region. In contrast, Asians have lower high-density lipoprotein-cholesterol (HDL-C) levels ${ }^{2}$. Furthermore, some countries including China, Spain, and the United States have reported a decrement of plasma lipid levels associated with a lesser number of cardiovascular deaths over the past two decades ${ }^{3}$. Therefore, periodical assessments of the contribution of modifiable and non-modifiable factors that influence plasma lipid levels at the national level are needed to design and adjust the cardiovascular risk reduction programs. This manuscript summarizes the epidemiology of dyslipidemia in Mexico. Our purpose is to highlight the peculiarities of our population regarding the clinical expression, genetics, and treatment of lipid disorders. Furthermore, it is a call for action to address the existing gaps in care and research of lipid disorders.

\section{EPIDEMIOLOGY OF DYSLIPIDEMIAS IN MEXICO}

In Mexico, non-communicable diseases (NCDs) have become the major health problem, with type 2 diabetes (T2D) and coronary heart disease as the leading causes of death in the adult population ${ }^{4}$. Contrary to what has been reported for other populations, Mexico has documented an increase in the prevalence and incidence of cardiovascular mortality and its main risk factors, including T2D, dyslipidemia, hypertension, overweight, and obesity, over the years. Probably, these data are influenced by the demographic transition that has occurred in our country in recent decades, but above all, they could be attributed to the change in lifestyle to a more sedentary one and to a shift in food consumption of high-energy content food and high amounts of refined carbohydrates and saturated fat.

In the past few decades, there have been numerous reports about the prevalence of lipid abnormalities in the Mexican population. National surveys are the main source of evidence. Mexico is among the few countries in the Americas that have updated the prevalence of NCDs and their risk factors regularly. Nationwide population-based health surveys have been performed with no difference in the inclusion or exclusion criteria between studies ${ }^{5-7}$. A progressive increment in the mean plasma cholesterol levels has been observed in the time period covered by the health surveys. In the first National survey, ENEC, conducted in 1993, the mean cholesterol levels were $182.7 \mathrm{mg} / \mathrm{dL}$; in the second one (ENSA, 2000), the mean value increased to $197.5 \mathrm{mg} / \mathrm{dL}$; and 6 years later, in ENSANUT 2006, the mean cholesterol level was $198.5 \mathrm{mg} / \mathrm{dL}$. Prevalence of hypercholesterolemia (defined as cholesterol $\geq 200 \mathrm{mg} / \mathrm{dL}$ ) changed from $27.1 \%$ in 1993 to $43.6 \%$ in 2006 , with an alarming growth registered in the younger subsets of the population. This rising trend was observed also for low-density lipoprotein cholesterol (LDL-C). Nearly 8 million Mexicans were candidates to pharmacological treatment to decrease their cardiovascular risk due to their LDL-C concentration ${ }^{8}$.

The abnormality most frequently described in Mexico is hypoalphalipoproteinemia ( $\mathrm{HDL}-\mathrm{C}<40 \mathrm{mg} / \mathrm{dL}$ ). It is present in $60.5 \%$ (95\% confidence interval $58.2-62.8 \%$ ) of the Mexican population, with a higher prevalence in men (68.1\% vs. $54.0 \%$ in 2006 ). The prevalence has remained unchanged in the 1993-2006 period, probably because of the strong genetic component that determines the HDL-C concentrations.

The prevalence of hypertriglyceridemia is higher in Mexico than that reported in the United States or Europe. In ENEC 1993, the mean triglyceride value was $213.4 \mathrm{mg} / \mathrm{dL}$, with $42.3 \%$ of the population having levels over $150 \mathrm{mg} / \mathrm{dL}$. In ENSA 2000, the mean triglyceride level was $181.7 \mathrm{mg} / \mathrm{dL}$, and finally, in ENSANUT 2006, this mean value decreased to $139.6 \mathrm{mg} / \mathrm{dL}$, with $31.5 \%$ of Mexican adults being diagnosed with hypertriglyceridemia. It has been observed that hypertriglyceridemia is more frequent in adults who live in metropolitan areas or in the central region of the country, without any influence being shown by educational level or socioeconomic status. It is well known that Hispanics have greater susceptibility to have hypertriglyceridemia 9 . For example, in the United States, the Hispanic population has a 
higher prevalence of hypertriglyceridemia $(40.4 \%$ for men and $31.6 \%$ for women) compared to Caucasians and African-Americans.

Prevalence of dyslipidemia (according to NCEP-ATPIII) in adults living in Mexico City and in other six Latin American cities was described in the Cardiovascular Risk Factor Multiple Evaluation in Latin America (CARMELA) study ${ }^{10,11}$. Notably, inhabitants of Mexico City had the highest prevalence for hypertriglyceridemia, with a mean plasma triglycerides concentration of $183.19 \mathrm{mg} / \mathrm{dL}$ ( $\mathrm{n}=1722$ Mexican adults). The prevalence of hypertriglyceridemia was higher in men $(43.3 \%)$ than in women $(23 \%)$. A clear age effect exists, showing a more atherogenic lipid profile in those individuals between 55 and 64 years old.

The Hispanic population has lower HDL cholesterol concentrations compared to other ethnic groups, even after adjusting for age, sex, waist circumference, body mass index, smoking status, and other possible confounding variables ${ }^{12}$. In this group, isolated hypoalphalipoproteinemia (low HDL-C with triglycerides in the normal range) is common. It is seen even in cases without any other metabolic syndrome traits.

The Mexican population has a remarkable social and ethnic diversity. Prevalence of dyslipidemia differs between the country's regions. The Northeast areas, especially in highly industrialized cities, have an elevated prevalence of cardiovascular disease and its risk factors (e.g., prevalence of hypercholesterolemia is $58.4 \%)^{13}$. The same trend has been found in children and adolescents ${ }^{3,14}$. In contrast, the southern states of Mexico have a low prevalence of hypercholesterolemia (i.e., similar to that reported nationwide in 1993); the highest prevalence of hypoalphalipoproteinemia ( $72 \%)$ is found in this area.

\section{GENETICS OF DYSLIPIDEMIA IN MEXICO}

Populations with Native American ancestry, including the Mexican Mestizo population, have an increased susceptibility to develop metabolic disorders, including dyslipidemias. Studies to identify the reasons for this trend are at an early stage. Regrettably, Hispanics have been underrepresented in the international consortia that have applied state-of-the-art technologies to study the pathogenesis of lipid disorders.
Genome-wide association studies (GWAS) and exome sequencing consortia have made possible the identification of more than 175 loci involved in lipoprotein metabolism ${ }^{15}$. Evidence in Mexican Mestizos is limited to two GWAS studies. These reports confirmed many of the associations informed in Caucasians and identified several ethnic-specific genetic variants (located in ABCA1, MLXIPL, APOA5, APOC3, LPL, SIK3, and RORA among others) that influence triglycerides and HDL-C concentrations ${ }^{9}$. These newly identified associations have been replicated by more than one group $^{16-18}$. Furthermore, using a trans-ethnic approach (e.g., cross-population allele screen [CPAS]) ${ }^{16}$, a large proportion of them has been confirmed. The best example of an ethnic-specific variant with a significant effect on plasma lipid levels is the R230C variant of $A B C A^{12,3}$. This variant is associated with a $30 \%$ decrease of the $A B C-A 1$ transporter, which plays a prominent role in HDL biogenesis. The R230C/ C230C risk genotype is common in Mayans (0.28), Purépechas (0.214), Yaquis (0.203), and Teeneks (0.179). It is absent in other ethnic groups (excepting some Asian populations). Its presence is associated with a mean decrement of $4 \%$ in $\mathrm{HDL}$ cholesterol concentrations; $12 \%$ of the HDL-C variance in the National Health Survey was explained by its presence. This variant is also associated with obesity. Ethnicspecific risk variants have been reported for other metabolic conditions (an SLC16A11 haplotype for T2D or rs738409 at PNPLA3 for non-alcoholic steatohepatitis) in populations with Native American background ${ }^{19}$. These groups include the Amerindian communities living in Canada and the United States, Mestizos living in the Latin American countries, and Hispanic residents living outside their countries. The Amerindians have suffered biological and human crises that have reshaped several times their environment, lifestyle, and the size of the population. Thus, this group has been under a selection process for centuries that may alter linkage disequilibrium patterns and change the frequency of rare variants with major effects.

\section{PRIMARY HYPERLIPIDEMIAS IN MEXICO}

Familial combined hyperlipidemia ( $F C H L)$ is the most frequent primary dyslipidemia, affecting $1-6 \%$ of the general population and $10-20 \%$ of subjects with premature coronary artery disease. It is characterized by 
a mild, fluctuating, and variable increase in plasma cholesterol, triglycerides, or both. The most distinctive feature of this disorder is the presence of high plasma levels of apolipoprotein B (apoB), defined by a plasma level above the $90^{\text {th }}$ population-specific percentile (108 mg/dL in Mexican men and $99 \mathrm{mg} / \mathrm{dL}$ in Mexican women). It is frequently associated with insulin resistance and the metabolic syndrome ${ }^{20}$. To date, the genetic cause of this condition is largely unknown. It is considered to be an oligogenic disorder with a complex mode of inheritance, since at least 3 chromosomal regions have been identified as responsible to increase the risk of suffering from this hyperlipidemia (1q21-23,11p, and 16q), but their relative contribution is still unknown ${ }^{20}$. The strongest association has been informed with a locus at 1q21-23, in which Upstream Transcription Factor 1 is the most likely candidate to explain it $^{21,22}$. In addition, our group identified two variants that modulate apoB concentrations in $\mathrm{FCHL}$ kindreds (rs1349411, located at $12 \mathrm{p} 13.31$, and rs1424032, located at $16 \mathrm{q} 21)^{23}$.

Familial hypercholesterolemia ( $\mathrm{FH})$ is the most common monogenic disorder that causes severe hypercholesterolemia. FH is characterized by moderateto-severe accumulation of LDL particles in plasma due to decreased LDL clearance secondary to mutations in either the $L D L$ receptor gene (LDLR), in $A P O B$, or in PCSK9. FH is a common cause of premature coronary artery disease, ectopic deposition of cholesterol (expressed as tendinous xanthomata), and premature cardiovascular death. A prevalence of $1 / 200-500$ is calculated for the heterozygous form of $\mathrm{FH}$ and 1/300,000-1,000,000 for the homozygous form, although recent data suggest that $\mathrm{FH}$ prevalence is greater than the originally considered. An FH Mexican national registry is an ongoing multicenter effort that will contribute to the characterization of $\mathrm{FH}$ in our country ${ }^{24}$. Two genetic studies have looked for the $\mathrm{FH}$ mutations in Mexicans. The most remarkable finding is that no mutation was observed in the $\mathrm{FH}$ genes in nearly $40 \%$ of the cases; this feature has been previously reported in other non-Caucasian populations. Among cases with identifiable changes, $L D L R$ mutations are the most common cause of $\mathrm{FH}$ in Mexico ${ }^{25,26}$. Four mutations had not been previously reported (Fs92ter108, C268R, Q718X, and Fs736ter743) and only one case had a mutation in $A P O B$ (R3500Q). Among the mutations in $L D L R$, some have been reported exclusively in Mexicans: c.682G>A (also referred as FH-Mexico), c.1055G>A (p.Cys352Tyr, FH-Mexico 2), and c.1090T>C (p.Cys364Arg, FHMexico 3$)^{27}$. To date, no $\mathrm{FH}$ case has been attributed to a PCSK9 mutation in Mexicans. Based on their prevalence and the large contribution that this condition has on the burden caused by cardiovascular disorders, a greater awareness and specific public policies against FH are needed. Universal coverage, cascade screening among first-degree relatives, and continuous access to high-intensity statin therapy have decreased the mortality of $\mathrm{FH}$ patients compared to that reported in the total population ${ }^{28}$. Here, we propose that $\mathrm{FH}$ should be included in the conditions covered by the Fund for the Protection against Catastrophic Expenses of the Popular Insurance ("Fondo de Protección contra Gastos Catastróficos," "Seguro Popular "). In addition, high-intensity statin therapy should be included in the drug catalog of every health system. These two actions will increase access to care and increase adherence to lipid-lowering therapy. This initiative is just an example of the work that is being done worldwide to improve and promote the recognition, diagnosis, and proper treatment of $\mathrm{FH}^{29}$.

\section{QUALITY OF CARE OF DYSLIPIDEMIAS IN MEXICO}

The main goal of the treatment of dyslipidemias is the prevention of cardiovascular events, which depends on the existence of prevention programs, the early detection of risk factors, access to lipid-lowering therapies, and the long-term achievement of treatment goals. In high-risk cases both, lifestyle changes and medications (i.e., statins) are required.

As it happens worldwide, dyslipidemias in Mexico are underdiagnosed and undertreated. Despite its high prevalence, only a small percentage of the affected subjects have been screened, are aware of their condition, and are under treatment, and their plasma lipid levels are in accordance with the treatment goal set in the national guidelines. In the CARMELA study, where the prevalence of dyslipidemia was sought in seven cities in Latin America, it was found that only $22 \%$ of previously diagnosed adults in Mexico City that qualified for having drug therapy was under active treatment ${ }^{11}$. 
A major challenge to be solved is to increase access to health care and structured treatment programs. Despite the great progress that has been made in recent years, Mexico continues to be a country with large disparities and inequality in access and use of health services. In 2001, the Mexican Government opened the Seguro Popular program, which aims to provide health-care services to the uninsured population and achieve the universal health coverage. Despite that, the percentage of the population that has had at least one plasma lipid measurement at least once in their lives remains low. In addition, screening programs are dissimilar between health-care systems. In many instances, random capillary cholesterol measurements are used, instead of the recommended fasting lipid profile ${ }^{30}$. Furthermore, a large proportion of the individuals with abnormal lipid levels is not included in treatment programs. Assessment of the overall cardiovascular risk and the identification of the cause(s) of dyslipidemia are rarely completed in primary care units. As a result, high- and low-risk cases are treated equally. In many instances, treatments are short-lived. Lack of awareness or motivation to receive treatment, no identification of the common barriers to achieve adherence to medical treatment, and insufficient access to lipid-lowering drugs are the most frequent reasons for premature suspension of the treatment. High-intensity statin therapy is not available in the majority of the public health systems.

Data published at the Mexican Non-Transmissible Diseases Observatory (Observatorio Mexicano de Enfermedades No Transmisibles [OMENT]) clearly show some of the challenges to be solved in the treatment of lipid disorders in public institutions in Mexico. The OMENT web page shows several sources of information due to the fragmented nature of our health system. Among 1,766,043 cases of chronic diseases treated at 12,424 primary care units coordinated by the Health Ministry, $27 \%$ had a cholesterol concentration $>200 \mathrm{mg} / \mathrm{dl}$. This percentage is lower than that reported in the general population; this observation suggests a high rate of missed diagnoses. Only $34.6 \%$ were treated for 4 or more years. Even more, only a small proportion of cases had statin therapy (13.2\%), being pravastatin the most frequently used (11.2\%). The pattern of care was the same in cases with or without cholesterol $>200 \mathrm{mg} / \mathrm{dl}$. These observations clearly show that treatment is not implemented following the current guidelines. A complimentary source of information available at the OMENT is the "Red de Excelencia en Diabetes" (Excellence in Diabetes Network). It contains data of 53,709 T2D cases treated at 137 primary care units. The prevalence of hypercholesterolemia (36\%) was lower than the reported in the general population. Nearly half of the cases had $\mathrm{LDL}-\mathrm{C}<100 \mathrm{mg} / \mathrm{dL}$, but the pattern of statin prescription (based on pravastatin) was the same regardless patients achieved or not the LDL-C target concentration. In summary, models of care for dyslipidemias in public health systems should be adapted to optimize the screening programs and the effectiveness of therapy.

Policies of the health insurance companies should be supervised by regulatory agencies to ensure that all high-risk cases have access to the lipid-lowering drugs that they need to achieve the treatment goals.

\section{CONCLUSIONS}

In Mexico, where cardiovascular diseases and diabetes are the leading causes of premature death, the study and treatment of dyslipidemias remain as an area of opportunity. The study of the pathophysiology of lipid disorders in the Mestizo population could lead to innovative research. The selection process that has suffered this ethnic group for centuries may explain their susceptibility for having metabolic disorders (i.e., low HDL-C concentrations, hypertriglyceridemia, abdominal obesity, and T2D) and the association of these conditions with ethnic-specific genetic variants. This racial group has been underrepresented in the majority of the international research consortia. On the other hand, despite the high prevalence of dyslipidemia in Mexican adults, there is a lack of awareness of these conditions. The public is not informed about the need for screening and the potential benefit of the lipid-lowering treatments. Patient-advocacy groups interested in lipid disorders are scant at an early stage of their development. Health professionals do not have enough training to do the differential diagnosis and for estimating the cardiovascular risk. Dyslipidemias are not among the priorities of the managers of the health systems in the prevention of cardiovascular disease; access to laboratory resources and medications is insufficient in primary care units despite the proven cost-benefit of the treatment of lipid disorders. Evidence-based public policies are 
needed to change the practice and allocation of assets in our health system to be capable of preventing cardiovascular diseases. In summary, this manuscript is a call for action to address the existing gaps in care and research of lipid disorders. Treatment of dyslipidemia should have a prominent role in any effort to decrease the number of preventable deaths caused by NCDs.

\section{REFERENCES}

1. Garcia-Garcia G, Aviles-Gomez R, Luquin-Arellano VH, et al. Cardiovascular risk factors in the Mexican population. Ren Fail. 2006;28:677-87.

2. Villarreal-Molina MT, Aguilar-Salinas CA, Rodríguez-Cruz M, et al. The ATP-binding cassette transporter A1 R230C variant affects HDL cholesterol levels and BMI in the Mexican population: association with obesity and obesity-related comorbidities. Diabetes. 2007;56:1881-7.

3. Villarreal-Molina MT, Flores-Dorantes MT, Arellano-Campos $\mathrm{O}$, et al. Association of the ATP-binding cassette transporter A1 R230C variant with early-onset Type 2 diabetes in a Mexican population. Diabetes. 2008;57:509-13.

4. Rojas R, Aguilar-Salinas CA, Jiménez-Corona A, et al. Metabolic syndrome in Mexican adults: results from the national health and nutrition survey 2006. Salud Publica Mex. 2010;52 Suppl 1:S11-8.

5. SSA. Encuesta Nacional de Enfermedades Crónicas 1993. México, D.F: Secretaría de Salud; 1994

6. Aguilar-Salinas CA, Olaiz G, Valles $\mathrm{V}$, et al. High prevalence of low HDL cholesterol concentrations and mixed hyperlipidemia in a Mexican nationwide survey. J Lipid Res. 2001;42:1298-307.

7. Aguilar-Salinas CA, Gómez-Pérez FJ, Rull J, et al. Prevalence of dyslipidemias in the Mexican national health and nutrition survey 2006. Salud Publica Mex. 2010;52 Suppl 1:S44-53.

8. Villalpando S, Shamah-Levy T, Rojas R, Aguilar-Salinas CA. Trends for Type 2 diabetes and other cardiovascular risk factors in Mexico from 1993-2006. Salud Publica Mex. 2010;52 Suppl 1:S72-9

9. Aguilar-Salinas CA, Tusie-Luna T, Pajukanta P. Genetic and environmental determinants of the susceptibility of Amerindian derived populations for having hypertriglyceridemia. Metabolism. 2014;63:887-94.

10. Escobedo-de la Peña J, de Jesús-Pérez R, Schargrodsky $\mathrm{H}$, Champagne B. Prevalencia de dislipidemias en la ciudad de México y su asociación con otros factores de riesgo cardiovascular. Resultados del estudio CARMELA. Gac Med Mex. 2014;150:128-36.

11. Vinueza R, Boissonnet $C P$, Acevedo $M$, et al. Dyslipidemia in seven Latin American cities: CARMELA study. Prev Med. 2010; 50:106-11.

12. Aguilar-Salinas C, Canizales-Quinteros S, Rojas-Martínez R, Mehta R, Villarreal-Molina M, Arellano-Campos O. Hypoalphalipoproteinemia in populations of native American ancestry: an opportunity to assess the interaction of genes and the environment. Curr Opin Lipidol. 2009;20:92-7.
13. Solís-Olivares CA Muñoz-Cardona M. Prevalencia de factores de riesgo cardiovascular en pacientes evaluados por dolor torácico. Rev Mex Cardiol. 2007;18:109-18.

14. Bibiloni MD, Salas R, De la Garza YE, et al. Serum lipid profile, prevalence of dyslipidaemia, and associated risk factors among northern Mexican adolescents. J Pediatr Gastroenterol Nutr. 2016;63:544-9.

15. Dron JS, Hegele RA. Genetics of triglycerides and the risk of atherosclerosis. Curr Atheroscler Rep. 2017;19:31.

16. Ko A, Cantor RM, Weissglas-Volkov D, et al. Amerindian-specific regions under positive selection harbour new lipid variants in Latinos. Nat Commun. 2014;5:3983

17. Suárez-Sánchez F, Klunder-Klunder M, Valladares-Salgado A, et al. APOA5 and APOA1 polymorphisms are associated with triglyceride levels in Mexican children. Pediatr Obes. 2017;12:330-6.

18. Parra EJ, Mazurek A, Gignoux CR, et al. Admixture mapping in two mexican samples identifies significant associations of locus ancestry with triglyceride levels in the BUD13/ZNF259/APOA5 region and fine mapping points to rs 964184 as the main driver of the association signal. PLoS One. 2017;12:e0172880.

19. Aguilar-Salinas CA Sevilla González MD Tusie-Luna MT Searching for the causal variants of the association between hypertriglyceridemia and the genome-wide association studies-derived signals? Take a look in the native American populations. Circ Cardiovasc Genet. 2017;10:pii:e002010.

20. Aguilar Salinas CA, Zamora M, Gómez-Díaz RA, et al. Familial combined hyperlipidemia: controversial aspects of its diagnosis and pathogenesis. Semin Vasc Med. 2004:4:203-9.

21. Huertas-Vázquez $\mathrm{A}$, del Rincón $\mathrm{J}$, Canizales-Quinteros $\mathrm{S}$, et al. Contribution of chromosome 1q21-q23 to familial combined hyperlipidemia in Mexican families. Ann Human Genet. 2004; 68:419-27.

22. Suviolahti E, Lilja HE, Pajukanta P. Unraveling the complex genetics of familial combined hyperlipidemia. Ann Med. 2006; 38:337-51.

23. Weissglas-Volkov D, Plaisier C, Huertas-Vázquez $A$, et al. Identification of two common variants contributing to serum apolipoprotein B levels in Mexicans. Arterioscler Thromb Vasc Biol. 2009;30:353-9.

24. Vallejo-Vaz AJ, Kondapally Seshasai SR, Cole D, et al. Familial hypercholesterolaemia: a global call to arms. Atherosclerosis. 2015:243:257-9.

25. Robles-Osorio L, Huerta-Zepeda A, Ordóñez ML, CanizalesQuinteros S, Díaz-Villaseñor A, Gutiérrez-Aguilar R, et al. Genetic heterogeneity of autosomal dominant hypercholesterolemia in Mexico. Arch Med Res. 2006:37:102-8.

26. Vaca G, Vázquez A, Magaña M, Ramírez M, Dávalos I, Martínez E. Mutational analysis of the LDL receptor and APOB genes in Mexican individuals with autosomal dominant hypercholesterolemia. Atherosclerosis. 2011;218:391-6.

27. Mehta R, Zubirán R, Martagón A, et al. The panorama of familial hypercholesterolemia in Latin America: a systematic review. ] Lipid Res. 2016:57:2115-29.

28. Santos R, Bourbon M, Alonso R, et al. Clinical and molecular aspects of familial hypercholesterolemia in Ibero-American countries. J Clin Lipidol. 2017;11:160-6.

29. Vallejo-Vaz A, Akram A, Seshasai SK, et al. Pooling and expanding registries of familial hypercholesterolaemia to assess gaps in care and improve disease management and outcomes: rationale and design of the global EAS familial hypercholesterolaemia studies collaboration. Atheroscler Suppl. 2016;22:1-32.

30. Rivera-Hernández M, Galárraga O. Type of insurance and use of preventive health services among older adults in Mexico. J Aging Health. 2015:27: 62-982. 